\title{
Accretion of a volatile-poor plume mantle from noble gas isotopes
}

\section{RITA PARAI}

Washington University in St. Louis

Presenting Author: parai@wustl.edu

Earth's mantle retains primordial volatiles acquired during accretion, and has evolved over time due to degassing, radiogenic production and regassing of surface volatiles through subduction. Professor Yuji Sano's foundational work on mantle volatile compositions and transport at subduction zones has elucidated the processes that shaped volatile budgets in terrestrial reservoirs today.

Mantle-derived samples from plume-influenced localities exhibit low ratios of radiogenic to primordial noble gas isotopes compared to mid-ocean ridge basalts (MORBs). The low radiogenic-to-primordial isotope ratios $\left(\right.$ e.g., $\left.{ }^{4} \mathrm{He} /{ }^{3} \mathrm{He},{ }^{21} \mathrm{Ne} /{ }^{22} \mathrm{Ne}\right)$ in plume mantle sources have been attributed to greater retention of primordial volatile budgets in the mantle reservoir tapped by plumes. A relatively undegassed plume mantle reflects a lesser extent of processing by partial melting and incorporation of recycled slabs.

Interpretation of heavier noble gas ( $\mathrm{Ar}, \mathrm{Xe})$ signatures is more complex due to regassing via subducted material, but low mantle source ${ }^{40} \mathrm{Ar} /{ }^{36} \mathrm{Ar}$ and fissiogenic Xe that is dominated by $\mathrm{Xe}$ from spontaneous fission of short-lived ${ }^{244} \mathrm{Pu}$ support a lessdegassed plume source [1,2]. However, the mantle plume source also exhibits a robust signature of regassed atmospheric Xe incorporation and fissiogenic $\mathrm{Xe}$ excesses comparable in magnitude to those determined for upper mantle sources [1-5]. These features of the plume Xe signature are challenging to reconcile with a gas-rich reservoir that has experienced limited mantle processing compared to the upper mantle.

Here I argue that the $\mathrm{Xe}$ isotopic signature of plumeinfluenced samples requires low Xe abundances in the ancient plume mantle relative to the ancient MORB source mantle at the end of accretion. The portion of the mantle sampled by plumes acquired a mixture of primordial volatiles reflecting accretion of volatile-poor solids in the presence of solar nebular gas and received limited contributions from volatile-rich accreted material compared to the upper mantle. A coupled model of He$\mathrm{Ne}-\mathrm{Xe}$ evolution is used to explore reservoir initial compositions, degassing and regassing histories.

[1] Mukhopadhyay (2012), Nature 486(7401), 101-104; [2] Petö et al. (2013), EPSL 369-370, 13-23; [3] Tucker et al. (2012), EPSL 355, 244-254; [4] Parai et al. (2012), EPSL 359, 227-239; [5] Bekaert et al. (2019), EPSL 525, 115766. 